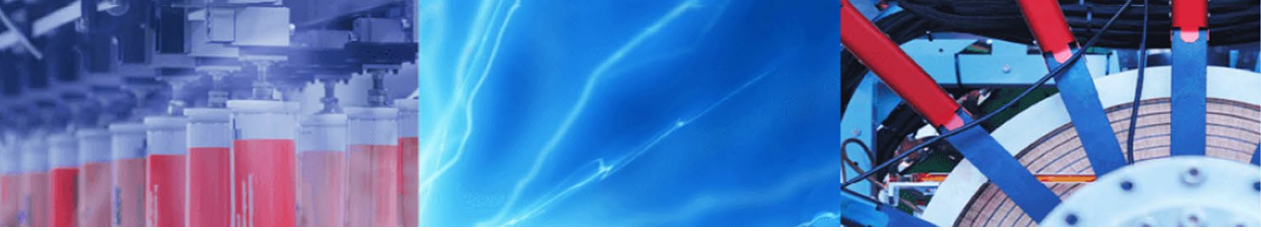

Research Article

\title{
Gradient field of undersea sound speed structure extracted from the GNSS-A oceanography: GNSS-A as a sensor for detecting sound speed gradient
}

\author{
Yusuke Yokota ${ }^{1}$ (i) $\cdot$ Tadashi Ishikawa $^{2}$
}

(c) Springer Nature Switzerland AG 2019

\begin{abstract}
The Global Navigation Satellite System-Acoustic ranging combination technique (GNSS-A) is a key observation method for detecting geodetic events such as co- and postseismic effects following a megathrust earthquake and the distributions of slip-deficit rate along the subduction zone. For capturing detailed slip and slip-deficit event information, Yokota et al. (Mar Geophys Res, 2018. https://doi.org/10.1007/s11001-018-9362-7) improved the accuracy of GNSS-A seafloor positioning using a method for extracting the gradient field of an undersea sound speed structure (SSS). In this paper, we examined how the extracted gradient parameters reflect a real ocean field. A comparison with the JCOPE2, a data assimilated ocean circulation model, shows that the extracted gradient parameters reflect the structure generated by a strong ocean current and can also be used for determination of the location and horizontal structure of the Kuroshio flow path. The results are also consistent with direct SSS observations. GNSS-A can thus serve as a sensor for detecting undersea sound speed gradient fields rather than surface condition.
\end{abstract}

Keywords GNSS-A - GNSS-A oceanography · Sound speed structure $\cdot$ Kuroshio

\section{Introduction}

The Global Navigation Satellite System (GNSS)-Acoustic ranging combination technique (GNSS-A) is a unique and important technology for detecting detailed absolute seafloor crustal deformation. Many key scientific findings have been obtained with the aid of GNSS-A [2-6]. Our data were published in Yokota et al. [7, 8].

The importance of various geophysical phenomena (e.g., postseismic mechanism, interseismic interplate coupling, and slow slip event) has been pointed out in seismology and disaster research [9]. The accuracy of GNSS-A is currently insufficient for detailed monitoring of these phenomena. Many GNSS-A studies have focused on undersea acoustic ranging to improve the accuracy [10-12]. Yokota et al. [1] proposed a technique for extracting the sound speed structure (SSS) through multiple modeling with simple time functions that depend on vessel and seafloor station positions. This technique not only improves the accuracy of seafloor position monitoring but also makes it possible to extract parameters considered to reflect the gradient state of the SSS.

In Yokota et al. [1], the verification of the extracted parameters was insufficient because only some of the data were compared with the ocean model. In this paper, we compare all extraction results obtained at sites HYG1 and HYG2 (located below the Kuroshio) with the JCOPE2, a data assimilated ocean circulation model constructed

Electronic supplementary material The online version of this article (https://doi.org/10.1007/s42452-019-0699-6) contains supplementary material, which is available to authorized users.

$\checkmark$ Yusuke Yokota, yyokota@iis.u-tokyo.ac.jp; Tadashi Ishikawa, ishikawa@jodc.go.jp| ${ }^{1}$ Institute of Industrial Science, The University of Tokyo, Tokyo, Japan. ${ }^{2}$ Hydrographic and Oceanographic Department, Japan Coast Guard, Tokyo, Japan.

SN Applied Sciences (2019) 1:693 | https://doi.org/10.1007/s42452-019-0699-6

Received: 27 February 2019 / Accepted: 30 May 2019 / Published online: 10 June 2019 


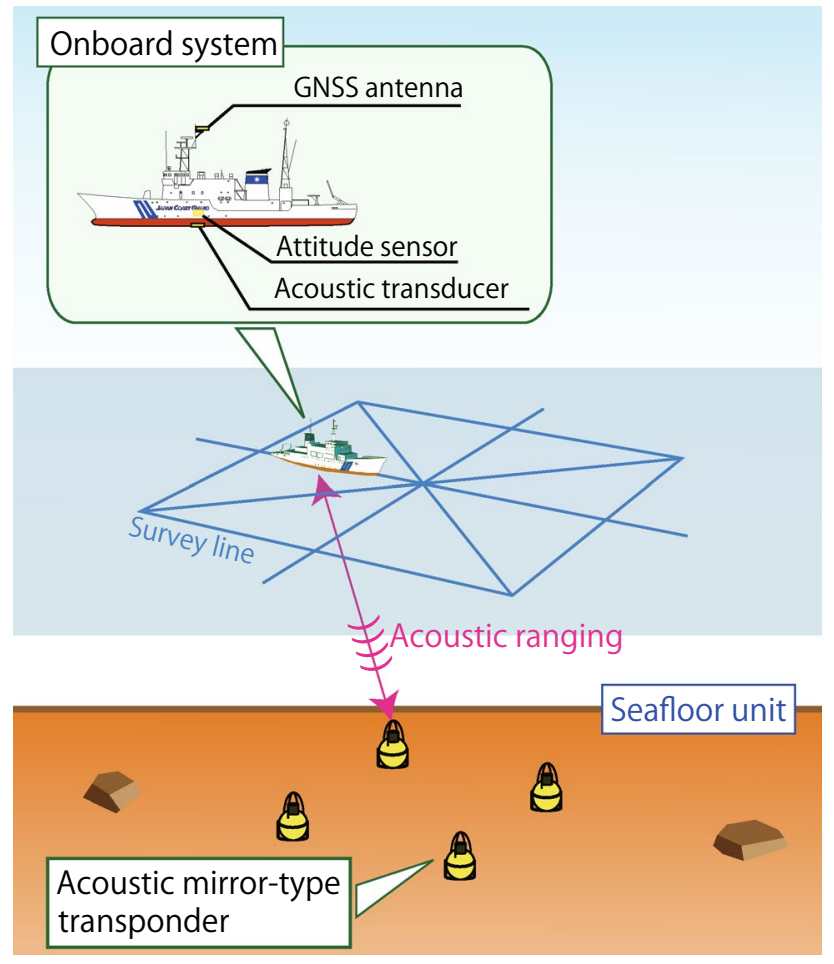

Fig. 1 Schematic diagrams of our seafloor geodetic observation system (modified after Asada and Yabuki [15] and Fujita et al. [18])

by Miyazawa et al. [13]. Additionally, the data on December 2017 are validated by comparing with in situ SSS measurements.

\section{Observation system}

GNSS-A, proposed by Spiess [14], determines seafloor position using a combination of GNSS data, obtained above the sea, and acoustic ranging data, obtained under the sea. Since the mid-1990s, we, the Hydrographic and Oceanographic Department, Japan Coast Guard (JHOD) and the Institute of Industrial Science (IIS), the University of Tokyo, have been developing a seafloor geodetic observation system based on this technique [15]. GNSS-A uses an ocean vessel as a relay point for GNSS observation and undersea acoustic ranging, as shown in Fig. 1. Four acoustic transponders are installed as seafloor stations at each site. The use of a vessel limits the observation frequency to about several times per year. The absolute position of a surface transducer is determined from attitude data and high-rate GNSS data analyzed using the software IT (Interferometric Translocation) [16, 17], and the distance between the vessel and a seafloor station is determined from acoustic ranging data based on travel time. The absolute position of the transducer and the relative distance

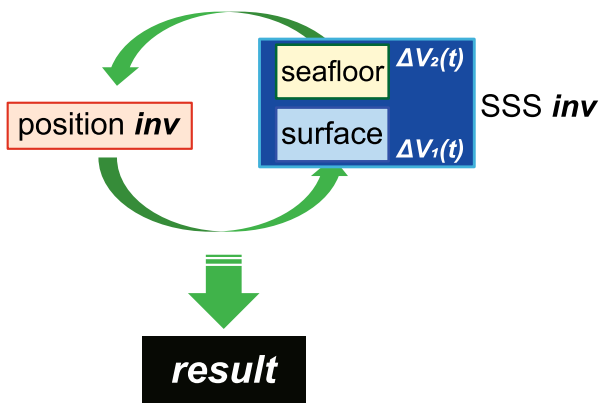

Fig. 2 Schematic diagram of inversion flow proposed by Yokota et al. [1]. A seafloor position inversion and SSS inversions are alternately iterated until the convergence to obtain the final solutions

data are combined to determine the absolute positions of the seafloor transponders.

We use survey lines composed of straight lines and a diamond around the site area. The straight lines are set so that the ship crosses the site area. For an overview of the SSS, sound speed depth profiles are acquired every few hours using temperature and salinity profilers, namely a conductivity temperature depth (CTD) profiler, expendable conductivity temperature depth (XCTD) profilers, and expendable bathythermographs (XBTs).

\section{SSS extraction method}

For determining the absolute seafloor positions, a linearized inversion based on the least squares formulation that combines the onboard transducer positions and the acoustic travel times is performed. We performed a position inversion and SSS inversions iteratively to obtain the final solutions in the inversion flow (Fig. 2). The SSS inversion method was developed by Yokota et al. [1] based on Fujita et al. [18] and Matsumoto et al. [19] and is described in detail in these papers. Here, we explain only the gradient parameters extracted from the SSS inversions.

In this method, large-scale temporal changes of SSS such as a daily change are first removed. Then, two gradient parameters are extracted from the inversion flow (Fig. 3). Primary gradient parameter $\Delta V_{1}(t)$ is extracted as a function of time $t$ dependent on vessel positions. As shown in Fig. 3a, acoustic signals are affected by a gradient field because they take different paths depending on the location of the vessel. For the left and right seafloor stations (Fig. 3a, b, respectively), the extracted $\Delta V_{1}(t)$ are almost the same since they depend on the area surrounded by the paths if the SSS is inclined linearly.

Secondary gradient parameter $\Delta V_{2}(t)$ is extracted as a function of time $t$ dependent on seafloor station positions. It reflects the difference in the acoustic paths due 


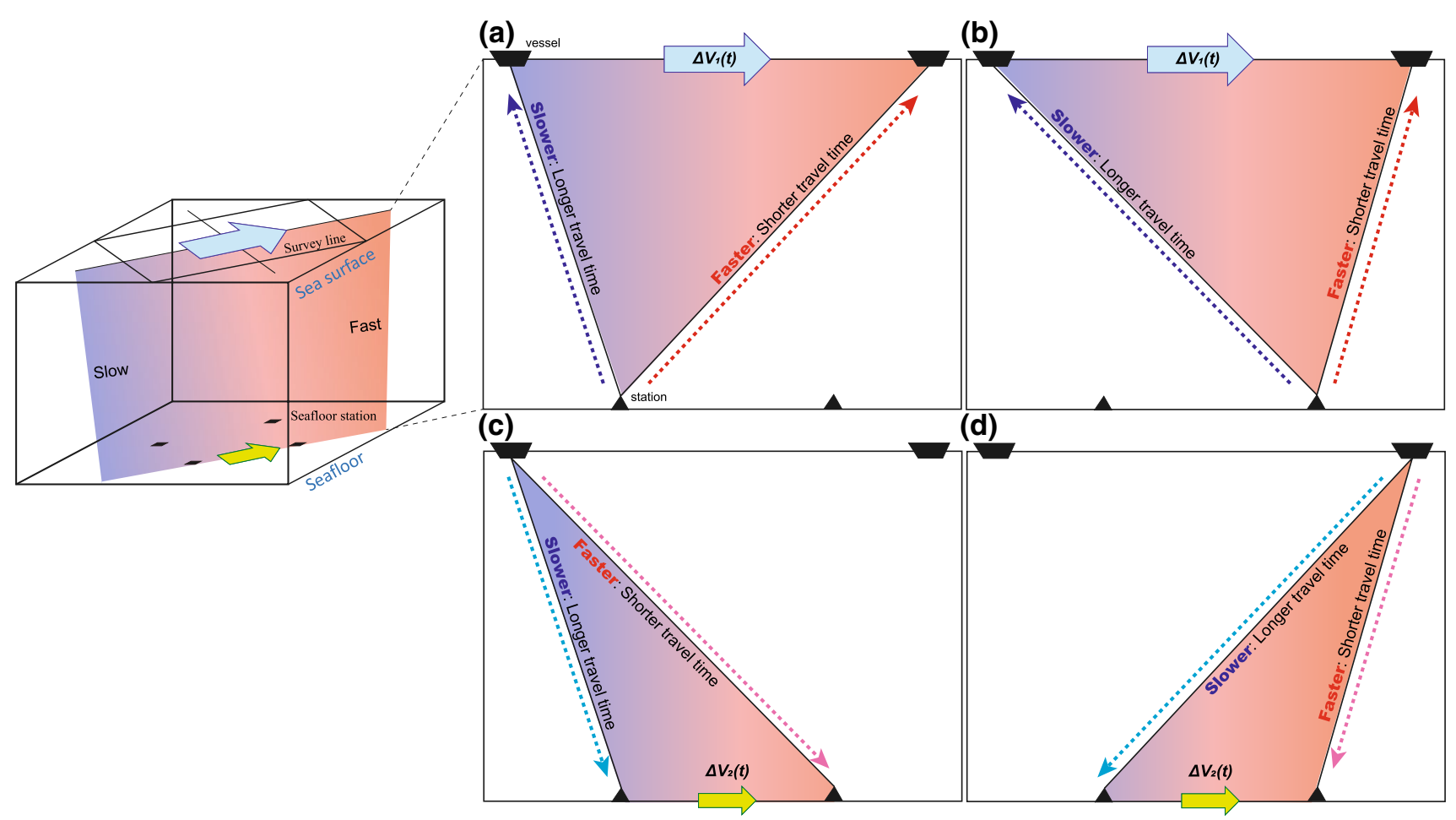

Fig. 3 Schematic cross-sectional views along a survey line for a rightward gradient field. Gradient parameter $\Delta V_{1}(t)$ extracted from surface vessel positions and $\mathbf{a}$ left and $\mathbf{b}$ right seafloor station data.

to the difference in the seafloor station position, as shown in Fig. 3c. Even when the vessel position and the scanned gradient field change (Fig. 3c, d), as is the case for a substantially linear gradient field, the extracted $\Delta V_{2}(t)$ in the cases of Fig. $3 c, d$ are almost the same except for a time change for the same reason given for $\Delta V_{1}(t)$.

$\Delta V_{1}(t)$ and $\Delta V_{2}(t)$ in this analysis are not set for each depth; instead, they are set to be the same for all depths. Therefore, the extracted components were normalized so that the gradient structure extends over the entire depth because the correction function is set to be the same for all depths. A gradient field cannot be correctly extracted with only one straight survey line, because this method has no resolution along the normal direction of a survey line. Therefore, we discuss only the values obtained by summarizing gradient parameters for multiple survey lines.

\section{Extracted parameters}

To discuss many cases, we compared the extracted parameters obtained at sites HYG1 and HYG2 (Fig. 4). These sites are, respectively, located east of the Hyuga-nada region and almost below the Kuroshio, which flows near the Japan Islands. Figure 5 shows all the extraction results between February 2012 and December 2017. As shown
Gradient parameter $\Delta V_{2}(t)$ extracted from seafloor station positions when the vessel is located in the $\mathbf{c}$ left and $\mathbf{d}$ right regions

in the lower-right example in Fig. 5, black and red vectors indicate extracted $\Delta V_{1}(t)$ and $\Delta V_{2}(t)$ parameters, respectively, normalized so that the gradient structure extends over the entire depth. Each arrow points in the direction of increasing sound speed (increasing temperature). As shown, the relationship between $\Delta V_{1}(t)$ and $\Delta V_{2}(t)$ varies. For example, for HYG1 (top graph in Fig. 5) in Epoch $13, \Delta V_{1}(t)$ is large and $\Delta V_{2}(t)$ is almost 0 , whereas for HYG2 (bottom graph in Fig. 5), both $\Delta V_{1}(t)$ and $\Delta V_{2}(t)$ are small, and their directions are different. These parameters are listed in Supplementary Material 1.

\section{Interpretation of extracted parameters}

We used a simple model to interpret the kind of undersea SSS expressed by the $\Delta V_{1}(t)$ and $\Delta V_{2}(t)$ parameters. Figure 6 shows sectional views of four cases for a survey line. In Case A, shown in Fig. 6a, the gradient field of the SSS is strong at the most disturbed surface layer. Since primary parameter $\Delta V_{1}(t)$ reflects the gradient between path-1 and path-3, it is strongly affected by the gradient. Secondary parameter $\Delta V_{2}(t)$ reflects only the gradient between path-1 and path-2, and thus, it is only slightly affected by the gradient. Figure $6 \mathrm{~b}$ shows Case $B$, for which the gradient field is located at a somewhat deep depth. 


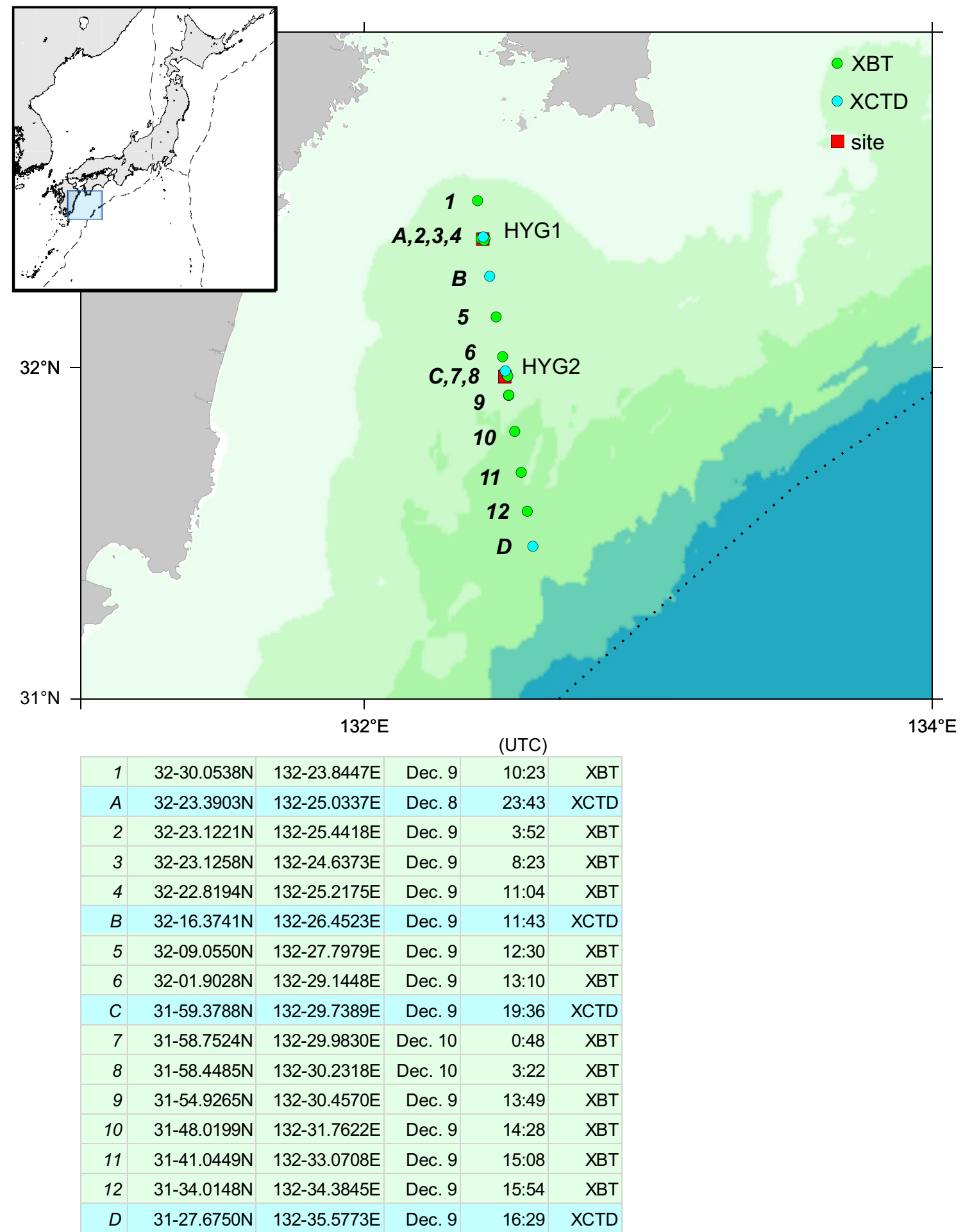

Fig. 4 (Upper) Locations of seafloor sites and direct sound speed observation points in December 2017. Red squares indicate seafloor sites HYG1 and HYG2. Green and light-blue circles indicate
XBT and XCTD observation points, respectively. (Bottom) Detailed information about XBT/XCTD casts is listed

In Case C (Fig. 6C), when the gradient field is located at a deep depth, $\Delta V_{2}(t)$ is larger than $\Delta V_{1}(t)$. For HYG1 and HYG2, this case was not observed. Case $D$ is much more 
(1) Jan. 27, 2012

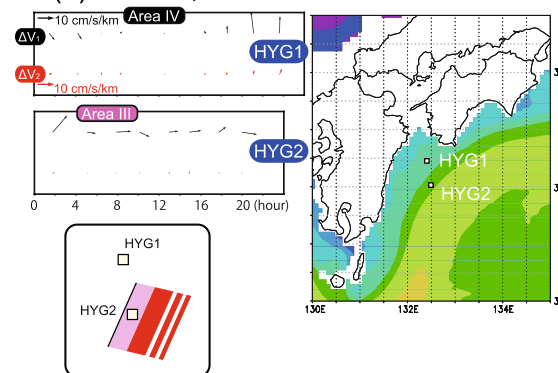

(4) Jan. 17, 2013
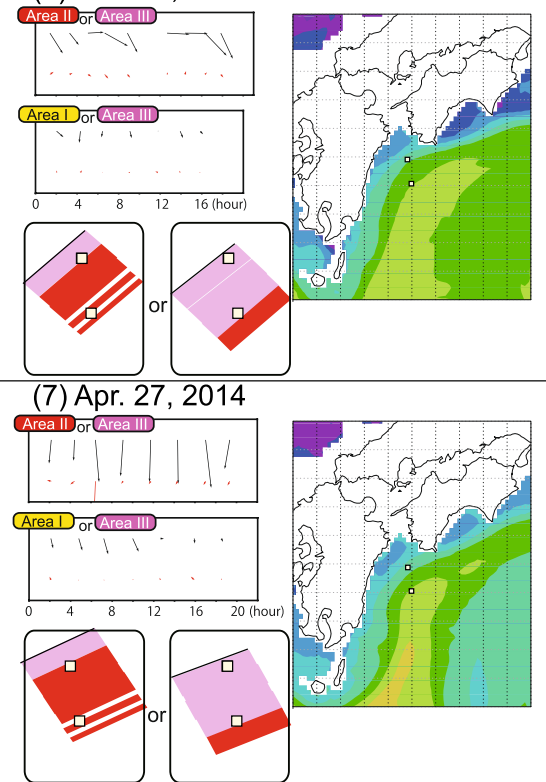

(10) May. 29, 2015

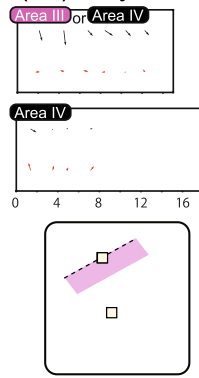

(13) Jan. 16, 2016
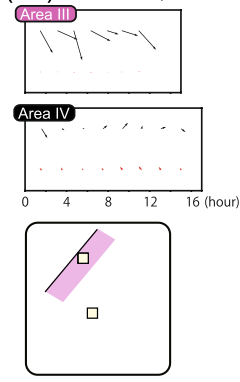

(2) Mar. 02, 2012

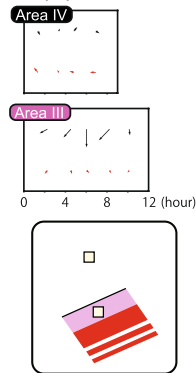

(5) Jul. 08, 2013
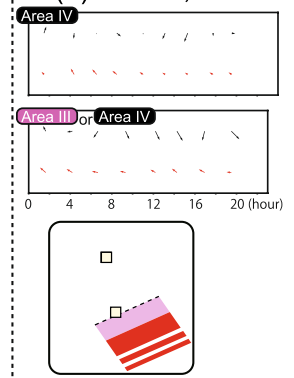

(8) Aug. 28, 2014

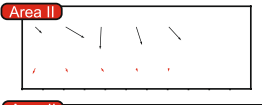

Areali
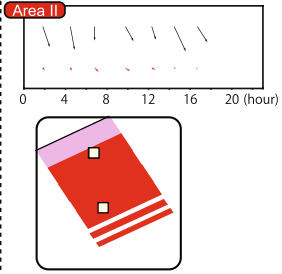

(11) Sep. 12, 2015
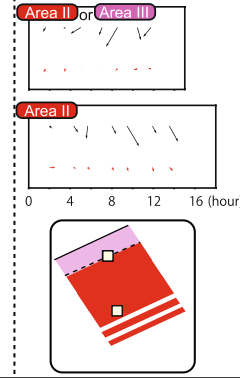

(14) Mar. 19, 2016

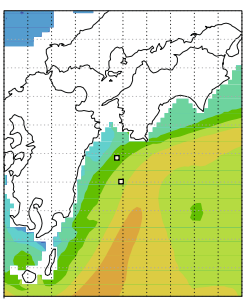

Area IV

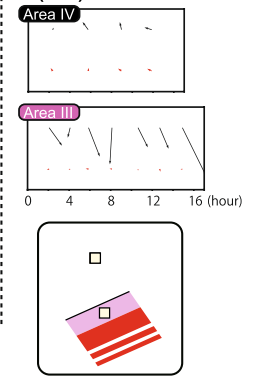

Fig. 5 Comparison between extracted gradient parameters at sites HYG1 and HYG2 and 100-m-deep temperature fields of the JCOPE2 model on observation dates for HYG2. Color scale of temperature fields is drawn on the right bottom. On the left shoulder of the graph for each gradient parameter, an area categorization (defined

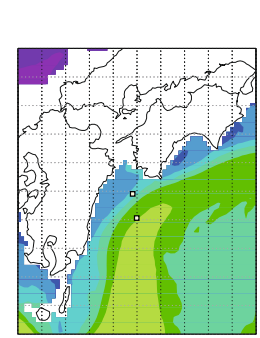

(3) Jun. 13,2012

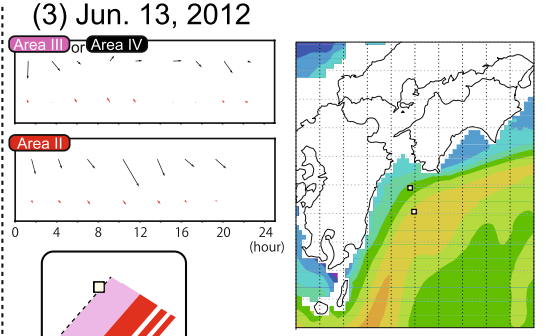

(6) Jan. 18, 2014
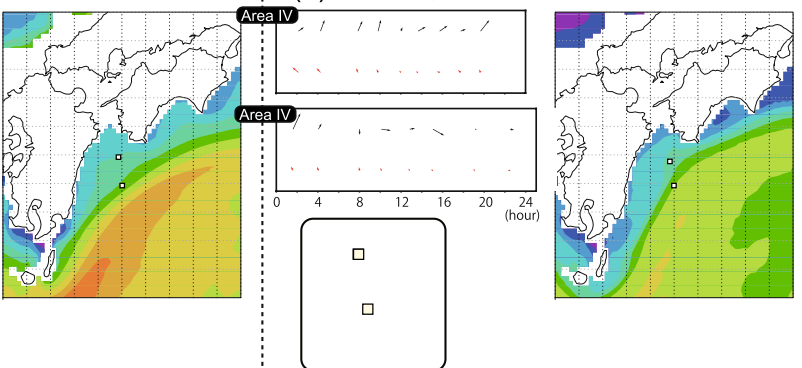

(9) Dec. 09, 2014
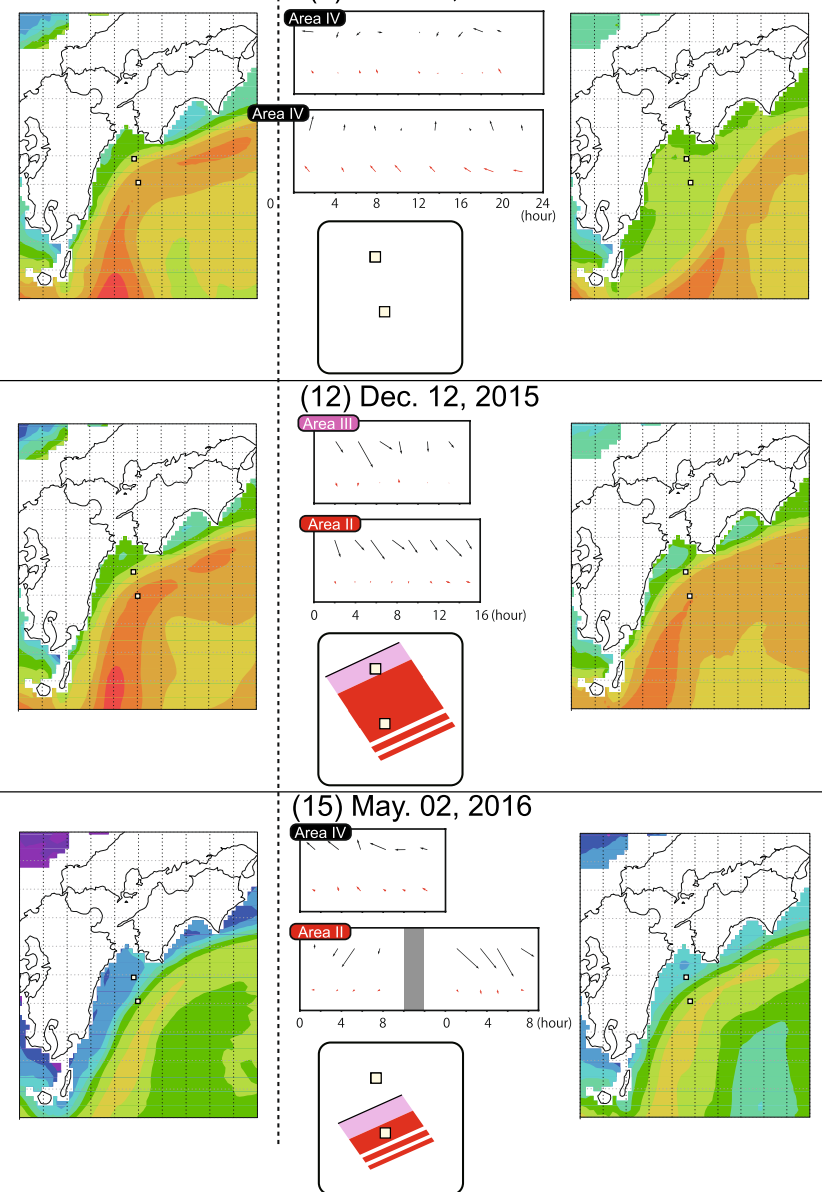

in Fig. 7) determined from the extracted parameters is shown. Based on these categorizations, an interpretation of the position and horizontal structure of the Kuroshio is shown at the bottom of the figure for each epoch 


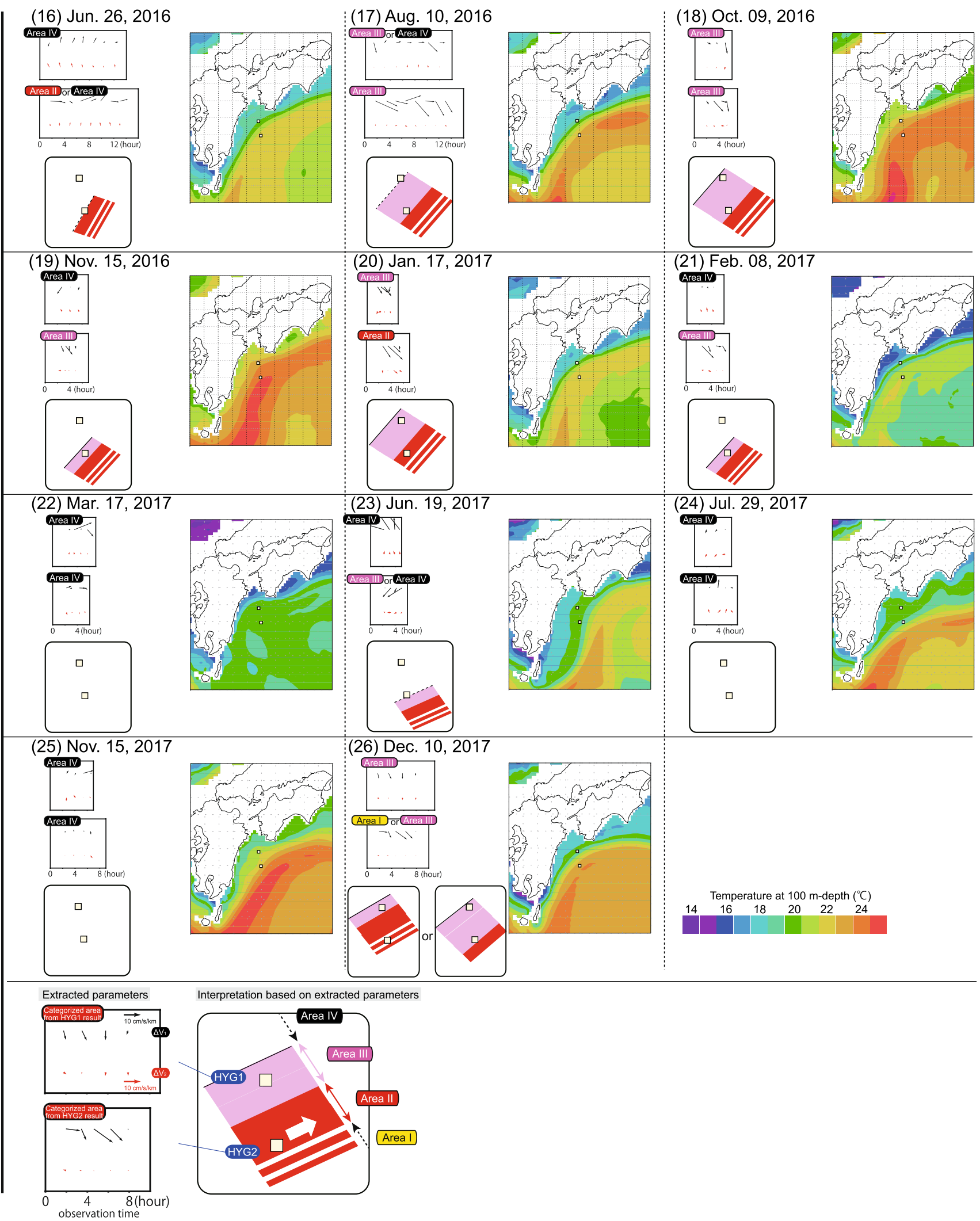

Fig. 5 (continued) 
(a)
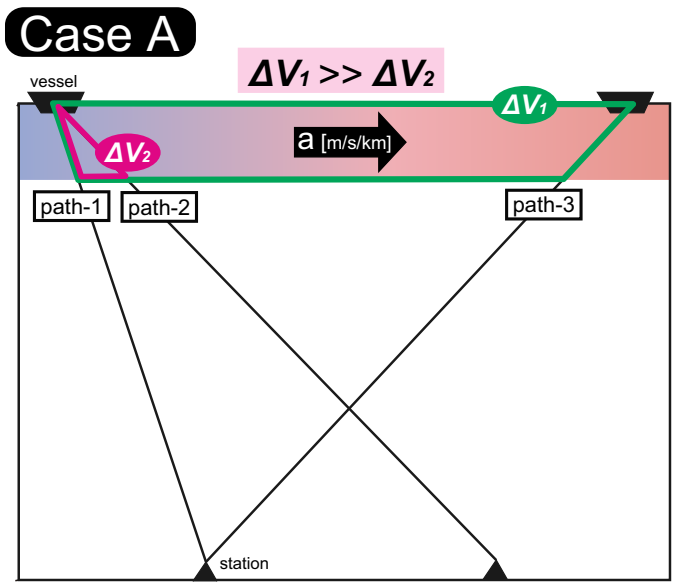

(c)

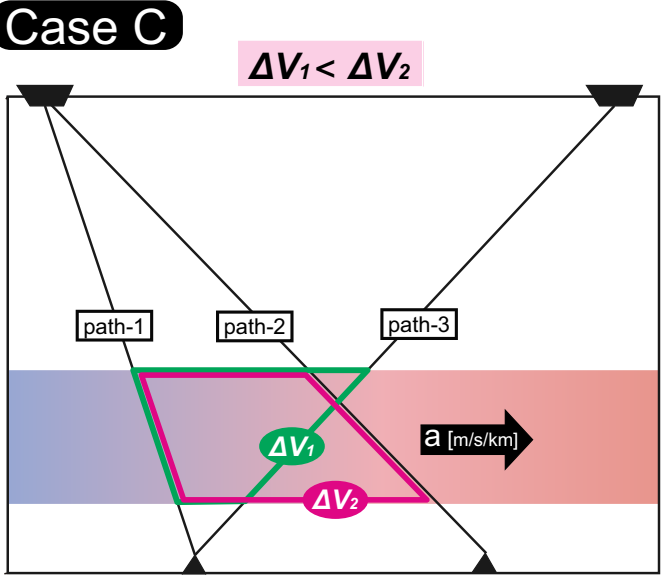

Fig. 6 Schematic diagrams of the relationships between extracted parameters and gradient SSSs. a Case A: there is a gradient field only in the shallow part. $\mathbf{b}$ Case B: there is a slightly deep and/or thick gradient field. c Case C: there is a gradient field in the deep-

complicated rather than the former cases (Fig. 6d). In such a gradient field, $\Delta V_{1}(t)$ is small compared to the absolute intensity of a shallow gradient field because it is affected by a reverse gradient field on the deep side. $\Delta V_{2}(t)$ may be largely affected by a deep gradient field in a direction different from that of the shallow gradient.

The cross section of the boundary region of the Kuroshio can be represented as shown in Fig. 7, since a gradient of SSS of the Kuroshio generally starts at the shallow region from the north ridge and progresses to the deep region as in the past direct XBT/CTD measurements [20, 21 ] and our direct measurements as shown in Fig. 8 a. The positional relationship between the site and the Kuroshio flow path can be divided into four areas. In the south region, Area I, there is stable flow, so it is unlikely that a disturbance like that in Case D will occur. The condition of the gradient field is thought to be similar to

\section{(b)}

\section{Case B}

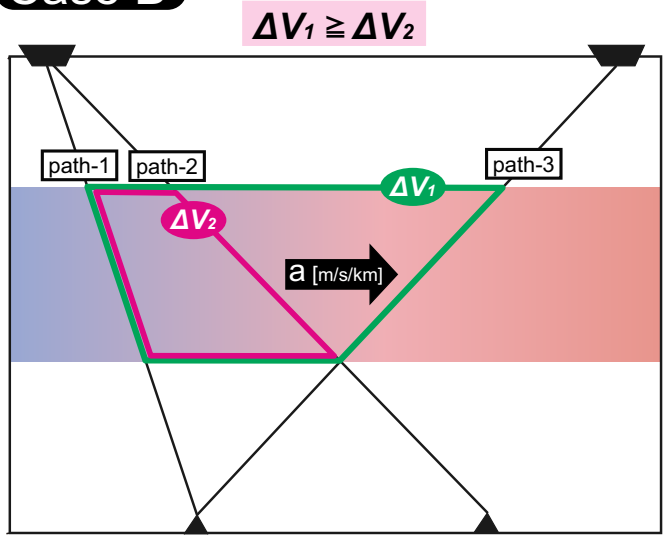

(d)

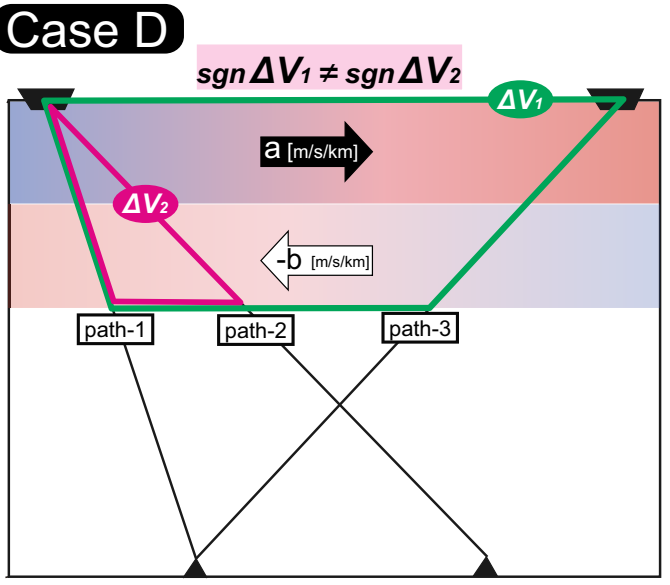

est part. $\Delta V_{1}(t)$ and $\Delta V_{2}(t)$ parameters give gradient fields in green regions surrounded by path- 1 and path- 3 and pink regions surrounded by path- 1 and path-2, respectively. $\mathbf{d}$ Case $D$ : there is a complicated gradient field

that for Case A, but the gradient parameters are small since there is no strong gradient field. In Area II, a thick gradient field is located in a deeper region, as in Case B. In Area III, there is only a shallow strong gradient field, as in Case A. In Area IV, outside the Kuroshio, unstable disturbance is likely to be generated, as in Case $D$, because there is no strong current flow. However, when the ocean field is stable, a weak gradient field may be generated, as in Case $A$, which has a small $\Delta V_{1}(t)$.

On the left shoulder of each graph in Fig. 5, an area categorization based on the extracted parameters is shown. The epochs that exhibited features of Cases B and $D$ were judged as Area II and Area IV, respectively. When $\Delta V_{1}(t)$ in a direction different from the Kuroshio flow region was extracted, the epochs were judged as Area IV. The epochs of Case A were judged as Area I when $\Delta V_{1}(t)$ was relatively small and as Area III when $\Delta V_{1}(t)$ 
Fig. 7 Schematic diagram of a north-south cross section of the SSS in the Kuroshio region. Area I indicates the south region inside the Kuroshio. Area II indicates the region that has a deep gradient in the Kuroshio. Area III indicates the region that has a shallow gradient in the Kuroshio. Area IV indicates the region outside the Kuroshio

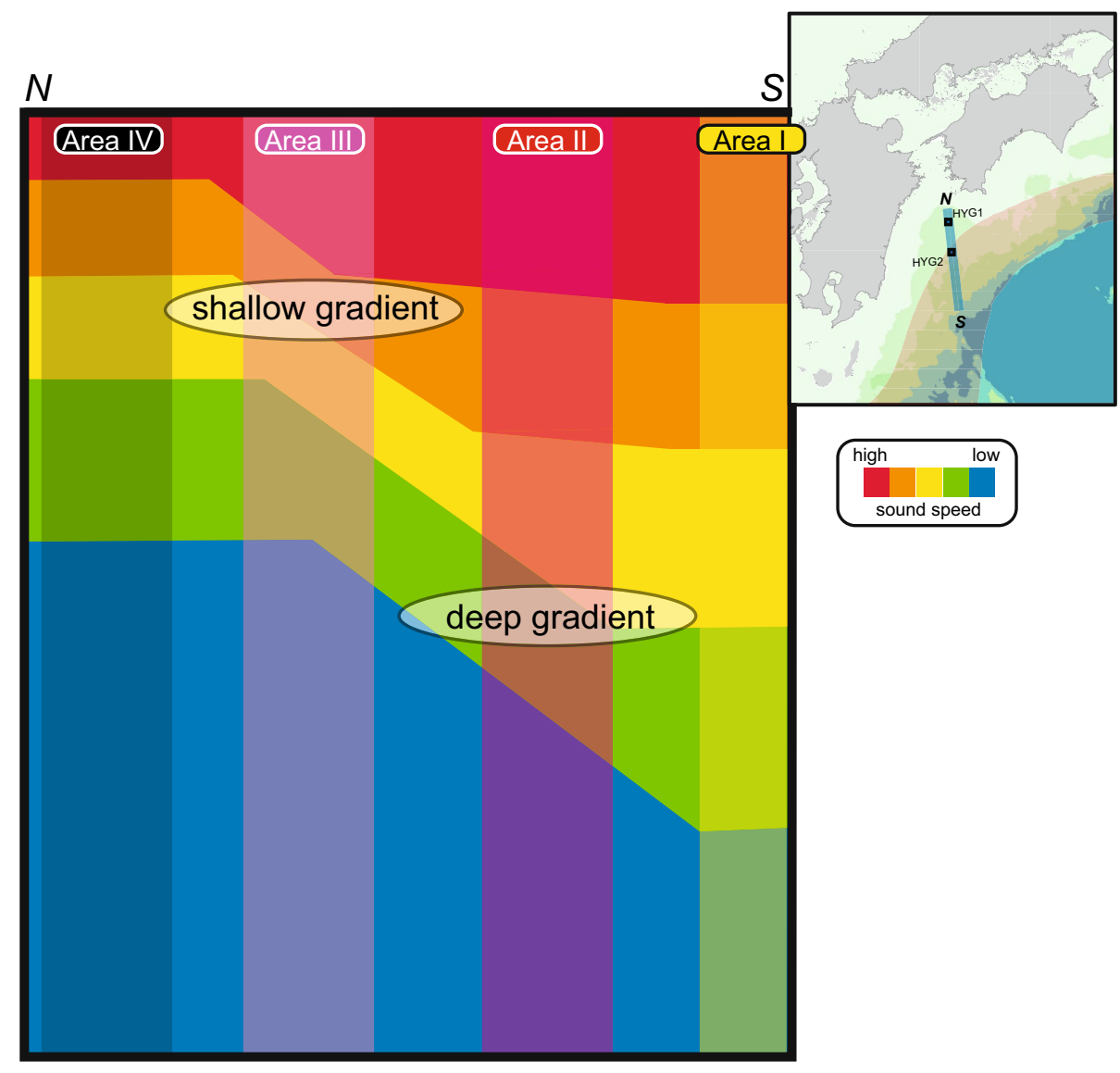

was relatively large. In other cases, the epochs were not determined and multiple possibilities are given. In order to verify the interpretation results, the temperature distribution at a depth of $100 \mathrm{~m}$ calculated from the JCOPE2 reanalysis model is also shown in each epoch in Fig. 5. These distributions are for HYG2 observation dates because HYG1 and HYG2 were always observed simultaneously.

\section{Interpretation: HYG2 results}

The HYG2 results (graphs in the second row in Fig. 5) for all the epochs can be identified as one of the four areas. For example, the results for Epochs 1, 2, 14, 17-19, and 21 can be categorized as Case $A$, because small $\Delta V_{2}(t)$ and large $\Delta V_{1}(t)$ parameters were extracted. Therefore, these epochs are judged to be Area III. The results for Epochs 3, 8, 11, 12, 15 , and 20 , in which $\Delta V_{2}(t)$ vectors are oriented in directions similar to those of $\Delta V_{1}(t)$ vectors, are categorized into Case B. Then, these epochs are judged to be Area II. In Epochs 9, $10,22,24$, and $25, \Delta V_{2}(t)$ are excessively large or their directions are different from those of $\Delta V_{1}(t)$. These epochs are categorized as Case D (Area IV). Some cases could not be categorized clearly. The parameters extracted in Epochs 5,
16 , and 23 have intermediate characteristics. Although the parameters extracted in Epochs 4, 7, and 26 can be categorized as Case $A$, since the field strength is small, area categorizations could not be made.

A comparison with the JCOPE2 model confirms that these categorizations are correlated with the positions of the Kuroshio flow path. In epochs categorized as Area III, the regions around site HYG2 are basically located northwest of the Kuroshio, which has a strong temperature gradient. In epochs categorized as Areas II and IV, the HYG2 site is basically inside the Kuroshio flow path and outside the Kuroshio, respectively.

\section{Interpretation: Kuroshio path investigated using HYG1 and HYG2}

We made a similar interpretation for HYG1 (graphs in the first column in Fig. 5). The positions and horizontal structures of the Kuroshio can be interpreted from the extracted SSS data at sites HYG1 and HYG2. In Fig. 5, based on the categorization for each epoch, the interpretation of the position and structure of the Kuroshio is shown at the bottom of the figure for each epoch. The meaning of the schematic interpretation image of the Kuroshio is 


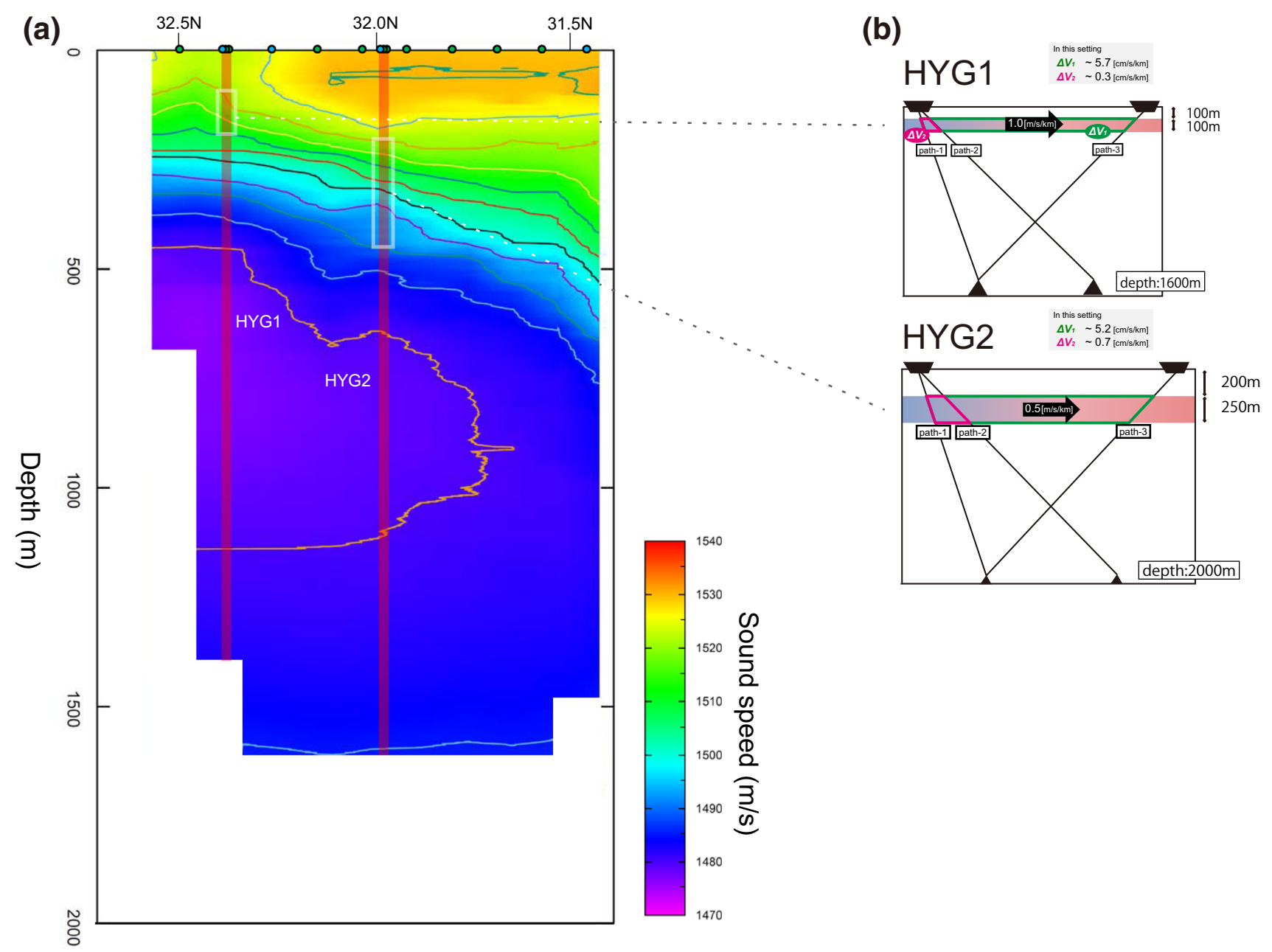

Fig. 8 Resultant SSS cross section of direct observations. Red bars indicate regions around sites HYG1 and HYG2. Green and blue circles at the surface indicate the position of XBT and XCTD casts, respectively. Contours are at every $5 \mathrm{~m} / \mathrm{s}$

explained in the lower-right example in Fig. 5. Each direction of flow was roughly interpreted from the directions of extracted parameters. For cases where the area categorization could not be completely discriminated due to an intermediate feature, the rough position of the northern bound and structure of the Kuroshio are shown (dotted line). For Epochs 4, 7, and 26, since there are multiple interpretations, multiple schematic images are shown.

First, we interpret the most characteristic cases. In the interpretations of Epochs 5, 6, 9, and 22-25, because the fields around HYG1 and HYG2 were unstable, the Kuroshio path is thought to be located in the offshore direction from these sites. The event in Epochs 22-25 was synchronized with the Kuroshio large meandering after 2017. In Epochs 1, 2, 14, 19, and 21, it can be interpreted that the Kuroshio was considerably offshore, although not as much as in the previous cases. Conversely, in Epochs 8 and 11, it can be interpreted that the Kuroshio flowed very near the coast. In Epochs 17 and 18, the results from both sites are categorized as Area III (or IV) and indicate the possibility that the flow of the Kuroshio was spread widely. In Epochs 10 and 13, although the HYG1 results were stable, the region around HYG 2 is believed to be located in an unstable field. The directions of $\Delta V_{2}(t)$ were also inconsistent with the steady state of the Kuroshio. It was thus inferred that the Kuroshio was meandering around HYG2 in each of these epochs. The interpretations for many epochs are mostly consistent with the JCOPE 2 model, suggesting that the extent of the Kuroshio berth can be determined from GNSS-A data.

Not all analysis results match the JCOPE2 model. In Epoch 16, $\Delta V_{2}(t)$ indicating the northward gradient different from $\Delta V_{1}(t)$ was estimated at HYG2. This is inconsistent with deeper parts of the JCOPE2 model. In Epochs 24 and 25 , although the gradient field is predicted to be located around the HYG2 site by the JCOPE2 model, this was not shown in the analysis results. These are not serious differences, and the results might complement the ocean 
model constructed based on existing data. For example, in Epochs 24 and 25, the actual Kuroshio flow path might have been located slightly south of the location predicted by the JCOPE2 model.

Narrow-scale (GNSS-A scale) sound velocity gradient fields in the Lesser Antilles were discussed in Sakic et al. [22] only using an opportunity measurement. The gradients for an order of magnitude between $1-10 \mathrm{~cm} / \mathrm{s} / \mathrm{km}$ estimated in that paper were similar with our results in Fig. 5. At least from this comparison, quantitatively similar gradient structure conditions were constructed in the undersea internal structures in the Lesser Antilles and the northwestern ridge of the Kuroshio.

For example, at HYG1 in Epoch 4 and at HYG2 in Epoch 8 , a half-day periodicity is visible in Fig. 5 . However, it cannot be determined whether this is, for example, a halfday SSS disturbance originating from the internal gravity wave or some error in the half-day period. A more detailed analysis of these gradient parameters will have to be performed in the future using more seafloor sites and data.

\section{Validation using direct observation}

Finally, we validated the results obtained GNSS-A data by a comparison with direct sound speed observations at a survey line that passes through seafloor sites HYG1 and HYG2 (Fig. 4). In an observation campaign in December 2017 (Epoch 26), we performed continuous XBT and XCTD observations along this survey line. Figure 8 a shows a cross section of the undersea SSS constructed from these observations.

As shown, the region around HYG1 was in a strong shallow (depth: 100-200 m) gradient field in the north region of the Kuroshio and is categorized as Area III. As shown in the results for Epoch 26 in Fig. 5, the extracted $\Delta V_{1}(t)$ and $\Delta V_{2}(t)$ parameters suggest Case $A$. This result is consistent with the direct SSS observation.

According to Fig. 8a, there was a thick gradient structure at a depth of around 400-700 m between HYG1 and HYG2. This region was categorized as Area II. The region around HYG2 had only a gentle weak gradient at a depth of around $200-400 \mathrm{~m}$ and was categorized as Area I. The extracted parameters were categorized as Case A, suggesting that area could be categorized Area I or Area III. This result is also consistent with the direct observation.

As shown in Fig. 8b, considering simple models, we can roughly estimate $\Delta V_{1}$ and $\Delta V_{2}$. Yokota [23] introduced this calculation method considering a ratio of areas. These results $\left(\Delta V_{1} \sim\right.$ about $5-6 \mathrm{~cm} / \mathrm{s} / \mathrm{km}$, and $\Delta V_{2} \sim$ about $\left.0-1 \mathrm{~cm} / \mathrm{s} / \mathrm{km}\right)$ are consistent to some extent with our estimation results $\left(\Delta V_{1} \sim\right.$ about $7-8 \mathrm{~cm} / \mathrm{s} / \mathrm{km}$, and $\Delta V_{2} \sim$ about $0-3 \mathrm{~cm} / \mathrm{s} / \mathrm{km}$ ) in Fig. 5 (Epoch 26 ) and suggest quantitatively the extraction ability for SSS of GNSS-A. $\Delta V_{2}$ of HYG1 result cannot be visible in the direct observation. It will have to be compared with more detailed monitoring in space and time to confirm it.

When seafloor position was modified largely, large $\Delta V_{2}$ was also extracted as shown in Yokota et al. [1]. On the other hand, even when large $\Delta V_{2}$ parameters were extracted, seafloor positions were not always largely modified. It indicates that there were datasets and environments where new method replaces $\Delta V_{1}$ with $\Delta V_{2}$ and seafloor position is not significantly modified. In this regard, statistical analysis with more data is needed in the future.

\section{Summary}

The results derived using the estimation method for a gradient SSS proposed by Yokota et al. [1] were found to be mostly in agreement with an ocean model (JCOPE2 model) and direct SSS observation. The extracted parameters can be used for determining the ocean field. These data can be used to monitor a much narrower region than that possible using other ocean observation techniques (e.g., satellite observation and direct buoy observation by Argo float). Although GNSS-A provides "fixed-point observations," "the gradient of SSS" can be detected. By using data from multiple sites, it is also possible to obtain a partial ocean structure.

GNSS-A can thus serve as a sensor for detecting undersea sound speed gradient fields. As a sensor with capabilities different from those of flow velocity sensors, such as current meter and the acoustic Doppler current profiler, and temperature and salinity sensors, such as XBT and CTD, GNSS-A can be used for investigating ocean current flow paths. Particularly, GNSS-A can be a sensor for undersea current structure rather than surface condition unlike a satellite observation. Temporally continuous (high-frequency) GNSS-A observation will make it possible to observe much more accurate SSS and seafloor position. The realization of a continuous GNSS-A monitoring system will contribute not only to earthquake disaster prevention research but also ocean research.

Acknowledgements We thank two anonymous reviewers for constructive comments. We would like to thank O. L. Colombo of the NASA Goddard Space Flight Center for providing us with the kinematic GNSS software IT (Interferometric Translocation) and the Geospatial Information Authority of Japan (GSI) for providing us with the high-rate GNSS data for the kinematic GNSS analysis and the daily coordinates of the sites on the GSI website. JCOPE2 data were obtained from the JAMSTEC's JCOPE website. We would also like to thank R. Zhang for lecturing and advising us about JCOPE2 data. Many members of the staff of the JHOD, including the crew of 
the survey vessels Takuyo, Shoyo, Meiyo, and Kaiyo, supported our observations and data processing.

\section{Compliance with ethical standards}

Conflict of interest The authors declare that they have no conflict of interest.

\section{References}

1. Yokota Y, Ishikawa T, Watanabe S (2018) Gradient field of undersea sound speed structure extracted from the GNSS-A oceanography. Mar Geophys Res. https://doi.org/10.1007/s1100 1-018-9362-7

2. Sato $M$, Ishikawa $T$, Ujihara $N$, Yoshida $S$, Fujita $M$, Mochizuki $M$, Asada A (2011) Displacement above the hypocenter of the 2011 Tohoku-oki earthquake. Science 332:1395. https://doi. org/10.1126/science.1207401

3. Watanabe S, Sato M, Fujita M, Ishikawa T, Yokota Y, Ujihara N, Asada A (2014) Evidence of viscoelastic deformation following the 2011 Tohoku-oki earthquake revealed from seafloor geodetic observation. Geophys Res Lett 41:5789-5796. https://doi. org/10.1002/2014GL061134

4. Yokota Y, Ishikawa T, Watanabe S, Tashiro T, Asada A (2016) Seafloor geodetic constraints on interplate coupling of the Nankai Trough megathrust zone. Nature 534:374-377. https://doi. org/10.1038/nature17632

5. Tomita F, Kido M, Ohta Y, linuma T, Hino R (2017) Along-trench variation in seafloor displacements after the 2011 Tohoku earthquake. Sci Adv 3:e1700113. https://doi.org/10.1126/sciad v.1700113

6. Tadokoro K, Nakamura M, Ando M, Kimura H, Watanabe T, Matsuhiro K (2018) Interplate coupling state at the Nansei-shoto (Ryukyu) trench, Japan, deduced from seafloor crustal information measurements. Geophys Res Lett 45:6869-6877. https:// doi.org/10.1029/2018GL078655

7. Yokota Y, Ishikawa T, Watanabe S (2018) Seafloor crustal deformation data along the subduction zones around Japan obtained by GNSS-A observations. Sci Data 5:180182. https:// doi.org/10.1038/sdata.2018.182

8. Yokota Y, Ishikawa T, Watanabe S (2018) Original data of seafloor crustal deformation along the subduction zones around Japanese Islands. PANGAEA. https://doi.org/10.1594/pangaea.88513 9

9. Obara K, Kato A (2016) Connecting slow earthquakes to huge earthquakes. Science 353:253-257. https://doi.org/10.1126/ science.aaf1512

10. Ikuta R, Tadokoro K, Ando M, Okuda T, Sugimoto S, Takatani K, Yada K, Besana GM (2008) A new GPS-acoustic method for measuring ocean floor crustal deformation: application to the Nankai Trough. J Geophys Res 113:B02401. https://doi. org/10.1029/2006JB004875

11. Kido $M$, Osada $Y$, Fujimoto $H$ (2008) Temporal variation of sound speed in ocean: a comparison between GPS/acoustic and in situ measurements. Earth Planets Space 60:229-234. https://doi. org/10.1186/BF03352785

12. Yasuda K, Tadokoro K, Taniguchi S, Kimura H, Matsuhiro K (2017) Interplate locking condition derived from seafloor geodetic observation in the shallowest subduction segment at the Central Nankai Trough, Japan. Geophys Res Lett 44:3572-3579. https://doi.org/10.1002/2017GL072918

13. Miyazawa $Y$, Zhang R, Guo X, Tamura H, Ambe D, Lee JS, Okuno A, Yoshinari H, Setou T, Komatsu K (2009) Water mass variability in the western north Pacific detected in a 15-year eddy resolving ocean reanalysis. J Oceanogr 65:737-756. https://doi. org/10.1008/s10872-009-0063-3

14. Spiess FN (1985) Suboceanic geodetic measurements. Geosci Remote Sens GE-23(4):502-510

15. Asada A, Yabiki T (2001) Centimeter-level positioning on the seafloor. Proc Jpn Acad Ser B 77:7-12. https://doi.org/10.2183/ plab.77.7

16. Colombo OL (1998) Long range kinematic GPS. In: Kleusberg A, Teunissen P (eds) GPS for Geodesy, 2nd edn. Springer, Berlin

17. Colombo OL, Sutter AW, Evans AG (2004) Evaluation of precise, kinematic GPS point positioning, In: Proceedings of ION GNSS2004, Long Beach, CA

18. Fujita $M$, Ishikawa $T$, Mochizuki $M$, Sato $M$, Toyama $S$, Katayama M, Kawai K, Matsumoto Y, Yabuki T, Asada A, Colombo OL (2006) GPS/acoustic seafloor geodetic observation: method of data analysis and its application. Earth Planet Space 58:265-275. https://doi.org/10.1186/BF03351923

19. Matsumoto Y, Fujita M, Ishikawa T (2008) Development of multiepoch method for determining seafloor station position (in Japanese, Abstract in English). Rep Hydro Ocean Res 26:16-22

20. Solomon $\mathrm{H}$ (1974) Observations of thermal microstructure in the Kuroshio off of southern Honshu and Shikoku. J Oceanogr Soc Jpn 30:108-120. https://doi.org/10.1007/BF02111109

21. Nagai T, Tandon A, Yamazaki H, Doubell MJ, Gallager S (2012) Direct observations of microscale turbulence and thermohaline structure in the Kuroshio front. J Geophys Res 117:C08013. https ://doi.org/10.1029/2011JC007228

22. Sakic P, Ballu V, Crawford WC, Wöppelmann G (2018) Acoustic ray tracing comparisons in the context of geodetic precise off-shore positioning experiments. Mar Geod 41(4):315-330. https://doi. org/10.1080/01490419.2018.1438322

23. Yokota Y (2019) Quantitative interpretation of the ability of the GNSS-A to monitor underwater structure (in Japanese, Abstract in English). J Mar Acoust Soc Jpn (in press)

Publisher's Note Springer Nature remains neutral with regard to jurisdictional claims in published maps and institutional affiliations. 